\title{
Trends, prevalence and factors associated with hypertension and diabetes among South African adults living with HIV, 2005- 2017
}

Nicola Chiwandire ${ }^{1 *}$, Nompumelelo Zungu ${ }^{2,3}$, Musawenkosi Mabaso ${ }^{2}$ and Charles Chasela ${ }^{1,4}$

\begin{abstract}
Background: Many people are now living longer with HIV due to access to antiretroviral treatment. In turn, there has been an increase in the burden of hypertension and diabetes. The paucity of data on the burden of hypertension and diabetes in adults living with HIV in South Africa is a public health concern. The paper aimed to describe the prevalence and factors associated with hypertension and diabetes among adults living with HIV (ALHI V).
\end{abstract}

Methods: This was a secondary data analysis of the population based on the South African National HIV Prevalence, Incidence, Behaviour and Communication surveys for 2005, 2008 and 2017. Descriptive statistics were used to summarise the characteristics of the study sample. Bivariate and multivariate logistic regression analyses were used to determine factors associated with hypertension and diabetes.

Results: The total study population of ALHIV aged 25 years and older was 978, 1023 and 2483 for 2005, 2008 and 2017. The prevalence of hypertension showed an increasing trend at $11.8 \%$ in 2005, 9.5\% in 2008 and $14.3 \%$ in 2017. The prevalence of diabetes was 3.3\% in 2005, 2.8\% in 2008 and 3.2\% in 2017. Increased odds of hypertension among adults living with HIV were consistently associated with being female and the age group 45 years older across all the survey years, including pensioners and the sick, living in urban areas, high risk of hazardous alcohol consumption, diabetes and heart disease. Increased odds of diabetes were consistently associated with hypertension across all the survey years, including age group 45 years and older, and poor health. While having a secondary level of education and above was protective against diabetes.

Conclusion: The study showed that the prevalence of hypertension is high and has increased over time among adults living with HIV while the prevalence of diabetes has remained constant. Findings identified factors consistently associated with the prevalence of both diseases overtime, including contemporary risk factors that should be targeted in the integrated management of chronic disease and HIV care model.

Keywords: Hypertension, Diabetes, HIV, South Africa, Prevalence, Factors, Trends

\footnotetext{
* Correspondence: nvchiwandire@gmail.com

'Division of Epidemiology and Biostatistics, School of Public Health, Faculty

of Health Sciences, University of the Witwatersrand, Johannesburg, South

Africa

Full list of author information is available at the end of the article
}

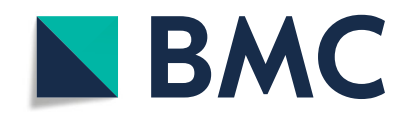

(c) The Author(s). 2021 Open Access This article is licensed under a Creative Commons Attribution 4.0 International License, which permits use, sharing, adaptation, distribution and reproduction in any medium or format, as long as you give appropriate credit to the original author(s) and the source, provide a link to the Creative Commons licence, and indicate if changes were made. The images or other third party material in this article are included in the article's Creative Commons licence, unless indicated otherwise in a credit line to the material. If material is not included in the article's Creative Commons licence and your intended use is not permitted by statutory regulation or exceeds the permitted use, you will need to obtain permission directly from the copyright holder. To view a copy of this licence, visit http://creativecommons.org/licenses/by/4.0/. The Creative Commons Public Domain Dedication waiver (http://creativecommons.org/publicdomain/zero/1.0/) applies to the data made available in this article, unless otherwise stated in a credit line to the data. 


\section{Background}

At 7.9 million, South Africa has the largest population of people living with HIV (PLHIV) in the world $[1,2]$. The successful roll-out of the anti-retroviral therapy (ART) programme since 2004 has contributed to improvements in the life expectancy and viral load suppression in PLHIV; and deaths due to opportunistic infections have also declined [3, 4]. This has transformed HIV infection into a chronic disease and evidence shows that as a consequence of a combination of factors including ageing, being on ART, HIV infection and certain lifestyle choices, their risk of developing non-communicable diseases (NCDs) including hypertension or diabetes increases [5]. In addition, the growing double burden of the HIV and NCD coepidemics has put significant pressure not only on the affected individuals but also on the struggling health system, existing health programmes and the economy in South Africa [6-10].

Studies that have been done to characterise the burden of hypertension and diabetes in PLHIV highlight the implication of not only collecting prevalence estimates but also adequately addressing multimorbidity in PLHIV and ensuring adequate availability of treatment to reduce the risk [7]. However, these studies present varied findings, suggesting that results and conclusions may not necessarily be extrapolated from one country to the next and not one intervention fits all [11-18]. A meta-analysis and systematic review across sub-Saharan Africa reported prevalence estimates for hypertension in PLHIV ranging between 5.2 and $50.0 \%$ and that of diabetes to range between 0.5 and $36.6 \%$. In South Africa, studies have also reported prevalence estimates in PLHIV for hypertension as high as $38.6 \%$ and diabetes as high as $8.66 \%[19,20]$. However, these estimates cannot be generalised to the entire South African population. Common risk factors of these NCDs in PLHIV have also been reported to include ageing, family history, use of ART, HIV infection, urbanisation, alcohol drinking and high body mass index in studies conducted in Ethiopia, Nigeria, Malawi, Tanzania, Zimbabwe and South Africa [13, 21-27]. Also, further studies indicated that hypertension and diabetes can also act as risk factors of each other [12].

Improved understanding of the magnitude of hypertension and diabetes and associated factors among PLHIV in South Africa is vital for informing public health interventions in the country. This paper investigates trends, prevalence and associated factors of hypertension and diabetes among adults living with HIV (ALHIV) in South Africa using the 2005, 2008, 2012, and 2017 nationally representative population-based HIV Prevalence, Incidence, Behaviour and Communication Surveys.

\section{Methodology}

\section{Study design and sample}

This secondary analysis utilised data collected from the South African National HIV Prevalence, Incidence, Behaviour, and Communication (SABSSM) surveys in 2005, 2008 and 2017. This was a multi-stage stratified cluster randomised cross-sectional population-based survey undertaken by the Human Sciences Research Council (HSRC). The details of the study and sampling method are described in detail elsewhere [1, 28-30]. Briefly, in the 2005 and 2008 surveys, in each household a maximum of three people were selected randomly to participate in the study, each representing the $2-14$ years, $15-24$ years and 25 years and older age groups [28, 29]. In the 2012 and 2017 surveys, all household members were eligible to participate in the survey $[1,30]$. The analysis for this paper was restricted to ALHIV aged 25 years and older who responded to the questions on the primary outcomes, hypertension and diabetes.

\section{Study variables}

The primary outcome variables were hypertension and diabetes. These variables were self-reported by the survey participants when asked the question "Do you currently have any of the following illnesses?". Both outcome variables were coded 1 for the answer "yes", and 0 for the answer "no". The explanatory variables used in this study were grouped into three categories namely socio-demographic, behavioural and healthrelated factors. The socio-demographic factors included sex (male and female), age (25-34 yrs., 35-44 yrs. and $45+$ yrs.), race (Non- Black African including White, Coloured and Indian; and Black African), marital status (never married and ever married), employment status (unemployed, employed and other (incl. Old age pensioner, sick/disabled and unable to work), educational level (no or up to primary and secondary and above), locality type (urban, urban formal, urban informal, tribal area/rural informal and rural formal) and province. The behavioural factors included hazardous alcohol consumption using the Alcohol Use Disorders Identification Test score (no risk (do not drink alcohol), low risk (0-7), medium risk (8-15), high risk (16-19) and addiction likely (20-40). Hazardous alcohol consumption was defined as a habit or amount of alcohol intake that raises the risk of adverse health effects [31]. The health-related factors included the exposure to ARVs (ARV exposed, ARV naive), viral load suppressed, perception of general health (excellent, good, fair, and poor), cancer, mental distress, tuberculosis, heart disease, diabetes and health care access (public care, and private care). 


\section{Statistical analysis}

All analyses were adjusted for complex survey design, clustering and any bias due to non-response using sample weights and the svyset and svy commands in STATA version 15 [32]. Frequency tables with percentages and median with interquartile ranges (IQR) were used to describe the characteristics of the study sample and trends in the prevalence of hypertension and diabetes in the study population. Pearson's chi-squared test was used for comparison of categorical variables. Bivariate logistic regression analysis was done and explanatory variables that had a $p$-value $<0.25$ were included in the multivariate logistic regression model to determine the associated factors. The Wald test was used to keep variables that had a $p$-value $<0.05$ through a process of removing, refitting, and verifying until all significant variables were kept in the final main effects model. Variables which had very wide confidence intervals were removed from the model regardless of the $p$-value. The Variance Inflation Factor test was done to check for multicollinearity at a cut off level of 5. A post-estimation test using the Hosmer-Lemeshow Goodness of Fit (GOF) test was done to test whether the final model was a good fit [33]. If the GOF test failed multiple times, the preliminary final model was used instead. Crude and adjusted odds ratios (aOR), 95\% confidence intervals $(\mathrm{CI})$ and $p$-values $\leq 0.05$ are reported to indicate the level of significance.

\section{Results}

\section{Trends in the prevalence of hypertension and diabetes}

A total of 978, 1023 and 2483 study participants aged 25 years and older were included for 2005, 2008 and 2017 respectively. Of these, the overall prevalence of hypertension was $11.8 \%$ in $2005,9.5 \%$ in 2008 and $14.3 \%$ in 2017. While the overall prevalence of diabetes was $3.3 \%$ in 2005, 2.8\% in 2008 and 3.2\% in 2017 (Tables 1 and 2). There was an increase in the trend of hypertension over the 3 years, whereas that of diabetes remained stagnant. The median age (IQR) for those who were hypertensive increased over the period from 37 (33-47) years in 2005, to $44(34-50)$ years in 2008 and 48 (3855) years in 2017 (Table 1). The median age for diabetes also increased from 35 (29-44) years in 2005 to 47 (3753) years in 2008 and 49 (42-57) years in 2017. Additionally, there was a decreased trend in hypertension and diabetes in the 25-34 age group over the 3 years, while the trend in the $45+$ years age group increased.

In 2005, 2008 and 2017 females had a higher prevalence of hypertension at 13.9, 12.8 and $16.6 \%$ respectively compared to males. Black Africans had the highest prevalence of hypertension at $11.8 \%$ in 2005 whereas, non-black Africans had the highest prevalence in 2008 and 2017 with 13.9 and $20.3 \%$ respectively. The prevalence estimates for diabetes were as follows: Black
Africans with $3.3 \%$ in 2005, Non-Black Africans with $8.0 \%$ in 2008 and Non-Black Africans with $5.6 \%$ in 2017. In 2017, those who were exposed to ARVs had a high prevalence of hypertension at $15.1 \%$ and a marginally lower prevalence of diabetes at $3.2 \%$ compared to those who were naïve to ARVs (Tables 1 and 2).

The prevalence distribution of hypertension was highest in the KwaZulu Natal province at 37.0\% in 2005 and for 2008 and 2017, the Gauteng province had the highest proportion of 27.6 and $30.4 \%$ respectively (Fig. 1).

The illustration shows that diabetes was highest in the KwaZulu Natal province in 2005 and 2008 at 25.5 and $28.2 \%$ respectively, and in 2017, the Gauteng Province had the highest estimation at $29.4 \%$ (Fig. 2).

\section{Factors associated with hypertension}

The factors associated with hypertension in 2005 included being female ( $\mathrm{aOR}=2.59 ; 95 \% \mathrm{CI}=1.26$ to 5.32 ), the age group $45+$ years $(\mathrm{aOR}=4.03 ; 95 \% \mathrm{CI}=1.96$ to $8.28)$, those with diabetes $(\mathrm{aOR}=11.73 ; 95 \% \mathrm{CI}=4.02$ to $34.20)$, and those with mental distress $(\mathrm{aOR}=2.97 ; 95 \%$ $\mathrm{CI}=1.59$ to 5.54 ) (Table 3). In 2008, factors associated with hypertension included being female $(\mathrm{aOR}=4.31$; $95 \% \mathrm{CI}=1.94$ to 9.58$)$, the age group $45+$ years $(\mathrm{aOR}=$ 8.44; $95 \% \mathrm{CI}=3.92$ to 18.15 ), those who perceived their health to be good ( $\mathrm{aOR}=3.72 ; 95 \% \mathrm{CI}=1.00$ to 13.81 ), those who perceived their health to be fair $(\mathrm{aOR}=5.44$; $95 \% \mathrm{CI}=1.40$ to 21.12 ), those who perceived their health to be poor ( $\mathrm{aOR}=4.82 ; 95 \% \mathrm{CI}=1.05$ to 22.20 ), those who lived in an urban formal area $(\mathrm{aOR}=2.95 ; 95 \% \mathrm{CI}=$ 1.34 to 6.53$)$, those with diabetes $(\mathrm{aOR}=6.16 ; 95 \% \mathrm{CI}=$ 1.68 to 22.59$)$ and those who accessed public health care services $(\mathrm{aOR}=2.58 ; 95 \% \mathrm{CI}=1.02$ to 6.51$)$ (Table 4$)$. While in 2017 factors associated with hypertension included being female ( $\mathrm{aOR}=2.33 ; 95 \% \mathrm{CI}=1.60$ to 3.42), the age group $45+$ years $(\mathrm{aOR}=7.32 ; 95 \% \mathrm{CI}=4.78$ to 11.21), pensioners and the sick $(\mathrm{aOR}=2.27 ; 95 \% \mathrm{CI}=$ 1.09 to 4.73$)$, those who lived in an urban area $(\mathrm{aOR}=$ $1.61 ; 95 \% \mathrm{CI}=1.16$ to 2.23 ), those who had a high risk of hazardous alcohol consumption $(\mathrm{aOR}=4.43 ; 95 \% \mathrm{CI}=$ 1.67 to 11.76$)$, those with diabetes $(\mathrm{aOR}=5.17 ; 95 \% \mathrm{CI}=$ 2.69 to 9.96$)$ and those with heart disease $(\mathrm{aOR}=3.36$; $95 \% \mathrm{CI}=1.59$ to 7.10$)$ (Table 5).

\section{Factors associated with diabetes}

The factors associated with diabetes in 2005 included being Black African ( $\mathrm{aOR}=5.70 ; 95 \% \mathrm{CI}=1.10$ to 29.44 ) and those with hypertension $(\mathrm{aOR}=11.71 ; 95 \% \mathrm{CI}=3.78$ to 36.29) (Table 6). In 2008, factors associated with diabetes included the age group $45+$ years $(\mathrm{aOR}=4.44 ; 95 \%$ $\mathrm{CI}=1.00$ to 19.73$)$, those who had hypertension $(\mathrm{aOR}=$ 7.56; $95 \% \mathrm{CI}=2.29$ to 24.92 ) and those who had heart disease $(\mathrm{aOR}=6.92 ; 95 \% \mathrm{CI}=1.62$ to 29.62$)$ (Table 7). While in 2017 factors associated with diabetes included 
Table 1 Sociodemographic characteristics and hypertension among ALHIV 25 years and older by survey year for 2005, 2008 and 2017 in South Africa

\begin{tabular}{|c|c|c|c|c|c|c|c|c|c|}
\hline \multirow[b]{2}{*}{$\begin{array}{l}\text { Population } \\
\text { characteristics }\end{array}$} & \multicolumn{3}{|l|}{2005} & \multicolumn{3}{|l|}{2008} & \multicolumn{3}{|l|}{2017} \\
\hline & Hypertension & $\begin{array}{l}\text { No } \\
\text { hypertension }\end{array}$ & $p$-value & Hypertension & $\begin{array}{l}\text { No } \\
\text { hypertension }\end{array}$ & $p$-value & Hypertension & $\begin{array}{l}\text { No } \\
\text { hypertension }\end{array}$ & $p$-value \\
\hline Overall & $126(11.8 \%)$ & 820 (88.2\%) & & 107 (9.5\%) & 859 (90.5\%) & & $372(14.3 \%)$ & 1955 (85.7\%) & \\
\hline \multicolumn{10}{|l|}{ Sex } \\
\hline Female & 97 (13.9\%) & $542(86.1 \%)$ & 0.057 & 93 (12.8\%) & $578(87.2 \%)$ & $<0.001$ & $289(16.6 \%)$ & 1365 (83.4\%) & $<0.001$ \\
\hline Male & 29 (8.2\%) & $278(91.8 \%)$ & & $14(3.0 \%)$ & 281 (97.0\%) & & $83(10.1 \%)$ & 590 (89.9\%) & \\
\hline \multicolumn{10}{|l|}{ Age } \\
\hline Median (IQR) & 37 (33-47) & 34 (29-39) & & $44(34-50)$ & 34 (29-39) & & $48(38-55)$ & $36(31-44)$ & \\
\hline 25-34 years & $30(8.6 \%)$ & $396(91.4 \%)$ & 0.009 & $21(4.9 \%)$ & 396 (95.1\%) & $<0.001$ & 48 (5.8\%) & 761 (94.3\%) & $<0.001$ \\
\hline $35-44$ years & 37 (12.0\%) & 288 (88.0\%) & & 31 (7.9\%) & 296 (92.1\%) & & 84 (9.8\%) & 647 (90.2\%) & \\
\hline $45+$ years & 59 (21.3\%) & $136(78.7 \%)$ & & 55 (26.1\%) & 167 (73.7\%) & & $240(30.6 \%)$ & $547(69.4 \%)$ & \\
\hline \multicolumn{10}{|l|}{ Race } \\
\hline Non-Black African & 15 (10.4\%) & 65 (89.6\%) & 0.757 & $4(13.9 \%)$ & $54(86.1 \%)$ & 0.476 & 41 (20.3\%) & 153 (79.7\%) & 0.087 \\
\hline Black African & $111(11.8 \%)$ & 754 (88.2\%) & & 103 (9.4\%) & 805 (90.6\%) & & $331(14.1 \%)$ & 1802 (85.9\%) & \\
\hline \multicolumn{10}{|l|}{ Marital status } \\
\hline Never married & 41 (9.5\%) & 437 (90.5\%) & 0.121 & $54(8.5 \%)$ & 524 (91.6\%) & 0.154 & $192(11.9 \%)$ & 1288 (88.1\%) & $<0.001$ \\
\hline Ever married & $84(14.3 \%)$ & $380(85.7 \%)$ & & $53(11.7 \%)$ & 332 (88.3\%) & & 180 (18.9\%) & 667 (81.1\%) & \\
\hline \multicolumn{10}{|c|}{ Highest educational qualification } \\
\hline None or Primary & $68(15.1 \%)$ & $315(84.9 \%)$ & 0.147 & $56(13.4 \%)$ & $274(86.6 \%)$ & 0.072 & $101(18.9 \%)$ & $441(81.1 \%)$ & 0.002 \\
\hline $\begin{array}{l}\text { Secondary and } \\
\text { above }\end{array}$ & $58(9.74 \%)$ & $503(90.3 \%)$ & & $50(8.0 \%)$ & $580(92.0 \%)$ & & $199(12.3 \%)$ & $1253(87.7 \%)$ & \\
\hline \multicolumn{10}{|l|}{ Employment status } \\
\hline Unemployed & $59(9.9 \%)$ & $462(90.1 \%)$ & 0.108 & $54(9.9 \%)$ & $423(90.1 \%)$ & 0.314 & $249(15.4 \%)$ & 1199 (84.6\%) & $<0.001$ \\
\hline Employed & $40(13.0 \%)$ & $291(87.0 \%)$ & & $34(7.9 \%)$ & $345(92.1 \%)$ & & 99 (11.0\%) & 718 (89.0\%) & \\
\hline Other & $26(20.3 \%)$ & $64(79.7 \%)$ & & $19(13.4 \%)$ & $90(86.6 \%)$ & & $23(33.7 \%)$ & $37(66.3 \%)$ & \\
\hline \multicolumn{10}{|l|}{ Locality } \\
\hline Urban formal & $57(12.7 \%)$ & $326(87.3 \%)$ & 0.491 & $47(12.4 \%)$ & $323(87.6 \%)$ & 0.072 & $236(15.9 \%)$ & 1027 (84.1\%) & 0.008 \\
\hline Urban informal & $34(14.2 \%)$ & $180(85.8 \%)$ & & $30(10.3 \%)$ & $208(89.7 \%)$ & & & & \\
\hline $\begin{array}{l}\text { Tribal area/Rural } \\
\text { informal }\end{array}$ & $28(10.7 \%)$ & $239(89.3 \%)$ & & $25(6.1 \%)$ & $238(93.9 \%)$ & & $99(11.6 \%)$ & $636(88.4 \%)$ & \\
\hline Rural formal & $7(6.2 \%)$ & 75 (93.8\%) & & $5(5.9 \%)$ & $90(94.1 \%)$ & & $37(10.1 \%)$ & $292(89.9 \%)$ & \\
\hline \multicolumn{10}{|c|}{ Hazardous alcohol consumption } \\
\hline No risk & $88(13.2 \%)$ & $558(86.8 \%)$ & 0.085 & $73(9.7 \%)$ & $574(90.3 \%)$ & & $261(14.1 \%)$ & 1359 (85.9\%) & \\
\hline Low risk & $24(6.7 \%)$ & $175(93.3 \%)$ & & $25(11.4 \%)$ & $181(88.6 \%)$ & 0.230 & $83(16.8 \%)$ & $407(83.2 \%)$ & 0.078 \\
\hline Medium risk & $12(16.4 \%)$ & $52(83.6 \%)$ & & $6(5.2 \%)$ & $62(94.8 \%)$ & & $13(7.1 \%)$ & 137 (92.9\%) & \\
\hline High risk & $1(5.5 \%)$ & $11(94.5 \%)$ & & $1(1.2 \%)$ & 15 (98.8\%) & & $8(22.6 \%)$ & $23(77.4 \%)$ & \\
\hline Addiction likely & $1(4.4 \%)$ & $11(95.6 \%)$ & & $1(1.7 \%)$ & $12(98.3 \%)$ & & $4(9.3 \%)$ & $25(90.7 \%)$ & \\
\hline \multicolumn{10}{|l|}{ Exposure to ARVs } \\
\hline ARV exposed & & & & & & & $234(15.1 \%)$ & 1150 (84.9\%) & 0.478 \\
\hline ARV naive & & & & & & & $107(13.0 \%)$ & $600(87.0 \%)$ & \\
\hline \multicolumn{10}{|l|}{ Viral load suppressed } \\
\hline Yes & & & & & & & $254(15.9 \%)$ & $1231(84.1 \%)$ & 0.033 \\
\hline No & & & & & & & $116(11.9 \%)$ & $703(88.1 \%)$ & \\
\hline
\end{tabular}


Table 1 Sociodemographic characteristics and hypertension among ALHIV 25 years and older by survey year for 2005, 2008 and 2017 in South Africa (Continued)

\begin{tabular}{|c|c|c|c|c|c|c|c|c|c|}
\hline \multirow[b]{2}{*}{$\begin{array}{l}\text { Population } \\
\text { characteristics }\end{array}$} & \multicolumn{3}{|l|}{2005} & \multicolumn{3}{|l|}{2008} & \multicolumn{3}{|l|}{2017} \\
\hline & Hypertension & $\begin{array}{l}\text { No } \\
\text { hypertension }\end{array}$ & $p$-value & Hypertension & $\begin{array}{l}\text { No } \\
\text { hypertension }\end{array}$ & $p$-value & Hypertension & $\begin{array}{l}\text { No } \\
\text { hypertension }\end{array}$ & $p$-value \\
\hline \multicolumn{10}{|c|}{ Perception of general health } \\
\hline Excellent & $5(7.3 \%)$ & $88(92.7 \%)$ & 0.003 & $5(2.2 \%)$ & $161(97.8 \%)$ & 0.006 & $42(10.0 \%)$ & $371(90.0 \%)$ & $<0.001$ \\
\hline Good & $55(9.0 \%)$ & $498(91.0 \%)$ & & $48(9.9 \%)$ & $444(90.1 \%)$ & & $186(13.2 \%)$ & $1134(86.8 \%)$ & \\
\hline Fair & $50(19.3 \%)$ & $182(80.7 \%)$ & & $43(14.5 \%)$ & $194(85.5 \%)$ & & $105(18.4 \%)$ & $370(81.6 \%)$ & \\
\hline Poor & $15(22.9 \%)$ & $51(77.1 \%)$ & & $11(10.0 \%)$ & $59(90.0 \%)$ & & $39(27.1 \%)$ & $78(72.9 \%)$ & \\
\hline \multicolumn{10}{|l|}{ Diabetes } \\
\hline Yes & $20(56.1 \%)$ & $14(43.9 \%)$ & $<0.001$ & $17(54.0 \%)$ & $10(46.0 \%)$ & $<0.001$ & $49(52.7 \%)$ & $35(47.3 \%)$ & $<0.001$ \\
\hline No & $103(10.3 \%)$ & $804(89.7 \%)$ & & $88(7.7 \%)$ & $849(92.3 \%)$ & & $322(13.1 \%)$ & 1919 (86.9\%) & \\
\hline \multicolumn{10}{|l|}{ Heart disease } \\
\hline Yes & & & & 7 (28.8\%) & $12(71.2 \%)$ & 0.008 & $23(41.8 \%)$ & $25(58.2 \%)$ & $<0.001$ \\
\hline No & & & & $99(8.7 \%)$ & $845(91.3 \%)$ & & $346(13.6 \%)$ & 1922 (86.4\%) & \\
\hline \multicolumn{10}{|l|}{ Cancer } \\
\hline Yes & $3(54.5 \%)$ & $2(45.5 \%)$ & 0.009 & $3(65.5 \%)$ & $3(34.5 \%)$ & $<0.001$ & $4(66.2 \%)$ & $4(33.8 \%)$ & $<0.001$ \\
\hline No & $120(11.5 \%)$ & 816 (88.5\%) & & $102(8.8 \%)$ & $855(91.2 \%)$ & & 365 (14.2\%) & $1946(85.8 \%)$ & \\
\hline \multicolumn{10}{|l|}{ Mental distress } \\
\hline Yes & $71(18.2 \%)$ & $327(81.8 \%)$ & 0.004 & $5(8.6 \%)$ & 31 (91.4\%) & 0.951 & $128(16.1 \%)$ & 512 (83.9\%) & 0.230 \\
\hline No & $53(7.52 \%)$ & 491 (92.5\%) & & 99 (8.9\%) & $826(91.1 \%)$ & & 244 (13.7\%) & $1443(86.3 \%)$ & \\
\hline \multicolumn{10}{|l|}{ Tuberculosis } \\
\hline Yes & $9(15.4 \%)$ & $54(84.6 \%)$ & 0.467 & $5(7.6 \%)$ & $44(92.5 \%)$ & 0.757 & $16(19.5 \%)$ & $60(80.5 \%)$ & 0.257 \\
\hline No & $114(11.5 \%)$ & $766(88.5 \%)$ & & $100(9.0 \%)$ & 815 (91.0\%) & & $352(14.1 \%)$ & 1891 (85.9\%) & \\
\hline \multicolumn{10}{|l|}{ Health care access } \\
\hline Public & 109 (12.7\%) & 675 (87.3\%) & 0.113 & $90(10.6 \%)$ & 649 (89.4\%) & 0.104 & $337(14.7 \%)$ & $1766(85.3 \%)$ & 0.296 \\
\hline Private & $15(7.1 \%)$ & 122 (92.9\%) & & $15(6.3 \%)$ & $186(93.7 \%)$ & & 35 (12.7\%) & 164 (87.3\%) & \\
\hline
\end{tabular}

the age group $45+$ years $(\mathrm{aOR}=7.90 ; 95 \% \mathrm{CI}=2.11$ to $29.58)$, those who reported having poor health $(\mathrm{aOR}=$ 6.48; $95 \% \mathrm{CI}=1.65$ to 25.41 ), those who had hypertension $(\mathrm{aOR}=4.60 ; 95 \% \mathrm{CI}=2.34$ to 9.07$)$, those who had secondary and above education $(\mathrm{aOR}=0.31 ; 95 \% \mathrm{CI}=$ 0.16 to 0.59 ) and those who accessed public health care $(\mathrm{aOR}=0.25 ; 95 \% \mathrm{CI}=0.11$ to 0.60$)$ (Table 8$)$.

\section{Discussion}

This study found an overall prevalence of hypertension among ALHIV in 2017 of 14.3\%, and this was an increase since 2005. This hypertension prevalence is comparable to other African studies that also report estimates ranging between 10.2 and $17.4 \%$ [12, 18, 34$36]$. On the other hand, the overall prevalence of diabetes was $3.2 \%$ in 2017, which remained the same as in 2005 and 2008. This prevalence is lower than previously reported in other African countries with estimates ranging between 5.6 and $8.66 \%$ [12-14, 20, 36, 37]. This may have been attributed to the participants not being aware of diabetes-related symptoms leading to the observed underestimation and this is supported by an IDF report stating that Africa has the highest proportion of undiagnosed diabetes [38]. Nevertheless, as PLHIV are more likely to interact with health facilities where hypertension screening is routine, case-finding and linkage to treatment can occur [39]. However different measures for diabetes will need to be implemented as routine vital checks at health facilities do not typically include diabetes tests unless patients have been recently diagnosed and appear "unwell" [39]. The findings also show that in 2005 most hypertensive and diabetic ALHIV were from the KwaZulu-Natal province. However, for 2017, the Gauteng province had the highest proportions of hypertensive and diabetic participants. Comparative studies to determine possible reasons for the shift from 2005 to 2017 could not be found at the time of this study. Nonetheless, according to the 2005 SABSSM survey, the KwaZulu-Natal province had a significantly higher number of PLHIV than the other provinces thus supporting our findings [28]. The shift in 2017 may have been due to urbanisation, 
Table 2 Sociodemographic characteristics and diabetes among ALHIV 25 years and older by survey year for 2005, 2008 and 2017 in South Africa

\begin{tabular}{|c|c|c|c|c|c|c|c|c|c|}
\hline \multirow[b]{2}{*}{ Population characteristics } & \multicolumn{3}{|l|}{2005} & \multicolumn{3}{|l|}{2008} & \multicolumn{3}{|l|}{2017} \\
\hline & Diabetes & No diabetes & $p$-value & Diabetes & No diabetes & $p$-value & Diabetes & No diabetes & $p$-value \\
\hline Overall & $34(3.3 \%)$ & 907 (96.7\%) & & $29(2.8 \%)$ & 937 (97.2\%) & & 87 (3.2\%) & $2242(96.8 \%)$ & \\
\hline \multicolumn{10}{|l|}{ Sex } \\
\hline Female & $22(2.5 \%)$ & $614(97.5 \%)$ & 0.198 & $26(3.5 \%)$ & 645 (96.5\%) & 0.227 & $63(3.6 \%)$ & $1592(96.4 \%)$ & 0.208 \\
\hline Male & $12(4.5 \%)$ & $293(95.5 \%)$ & & $3(1.5 \%)$ & $292(98.5 \%)$ & & $24(2.5 \%)$ & $650(97.5 \%)$ & \\
\hline \multicolumn{10}{|l|}{ Age } \\
\hline Median (IQR) & $35(29-44)$ & $34(29-40)$ & & $47(37-53)$ & $34(29-40)$ & & $49(42-57)$ & $37(32-46)$ & \\
\hline $25-34$ years & $10(3.1 \%)$ & 415 (96.9\%) & 0.866 & $5(1.1 \%)$ & 411 (98.9\%) & $<0.001$ & $4(0.7 \%)$ & 804 (99.3\%) & $<0.001$ \\
\hline $35-44$ years & $12(3.1 \%)$ & 312 (96.9\%) & & $9(2.2 \%)$ & 317 (97.8\%) & & $21(2.8 \%)$ & 710 (97.2\%) & \\
\hline $45+$ years & $12(4.1 \%)$ & $180(95.9 \%)$ & & $15(9.2 \%)$ & 209 (90.8\%) & & $62(6.8 \%)$ & $728(93.2 \%)$ & \\
\hline \multicolumn{10}{|l|}{ Race } \\
\hline Non-Black African & $3(0.9 \%)$ & $76(99.1 \%)$ & 0.030 & $3(8.0 \%)$ & $55(92.0 \%)$ & 0.103 & $10(5.6 \%)$ & $183(94.4 \%)$ & 0.184 \\
\hline Black African & $31(3.3 \%)$ & $830(96.7 \%)$ & & $26(2.7 \%)$ & $882(97.3 \%)$ & & $77(3.1 \%)$ & $2059(96.9 \%)$ & \\
\hline \multicolumn{10}{|l|}{ Marital status } \\
\hline Never married & $11(3.2 \%)$ & $465(96.8 \%)$ & 0.944 & $11(2.0 \%)$ & $566(98.0 \%)$ & 0.097 & $38(2.8 \%)$ & $1441(97.2 \%)$ & 0.250 \\
\hline Ever married & $23(3.3 \%)$ & $438(96.7 \%)$ & & $18(4.5 \%)$ & $368(95.5 \%)$ & & 49 (3.9\%) & $801(96.1 \%)$ & \\
\hline \multicolumn{10}{|l|}{ Highest educational qualification } \\
\hline None or Primary & $12(3.0 \%)$ & $370(97.0 \%)$ & 0.915 & $12(4.5 \%)$ & $318(95.5 \%)$ & 0.157 & $34(7.1 \%)$ & 509 (92.9\%) & $<0.001$ \\
\hline Secondary and above & $22(3.5 \%)$ & $535(96.5 \%)$ & & $16(2.2 \%)$ & $614(97.9 \%)$ & & $37(1.7 \%)$ & $1416(98.3 \%)$ & \\
\hline \multicolumn{10}{|l|}{ Employment status } \\
\hline Unemployed & $17(3.0 \%)$ & $501(97.0 \%)$ & 0.915 & $17(2.4 \%)$ & $460(97.6 \%)$ & 0.528 & $56(3.4 \%)$ & $1394(96.6 \%)$ & 0.582 \\
\hline Employed & $13(3.7 \%)$ & 317 (96.3\%) & & $9(2.9 \%)$ & 370 (97.1\%) & & $29(3.0 \%)$ & $788(97.0 \%)$ & \\
\hline $\begin{array}{l}\text { Other (incl. Old age pensioner. } \\
\text { Sick/disabled and unable to work) }\end{array}$ & $4(3.4 \%)$ & $85(96.6 \%)$ & & $3(5.0 \%)$ & $106(95.0 \%)$ & & $2(1.4 \%)$ & $58(98.6 \%)$ & \\
\hline \multicolumn{10}{|l|}{ Locality } \\
\hline Urban formal & $18(4.2 \%)$ & $361(95.8 \%)$ & 0.271 & $11(3.1 \%)$ & 359 (96.9\%) & 0.797 & $56(3.5 \%)$ & $1209(96.5 \%)$ & 0.241 \\
\hline Urban informal & $10(4.8 \%)$ & $203(95.2 \%)$ & & $6(2.0 \%)$ & $231(98.0 \%)$ & & & & \\
\hline Tribal area/Rural informal & $6(2.1 \%)$ & $261(97.9 \%)$ & & $9(2.5 \%)$ & 255 (97.53\%) & & $24(2.8 \%)$ & 711 (97.2\%) & \\
\hline Rural formal & $0(0.0 \%)$ & $82(100 \%)$ & & $3(4.6 \%)$ & $92(95.4 \%)$ & & $7(1.4 \%)$ & $322(98.6 \%)$ & \\
\hline \multicolumn{10}{|l|}{ Hazardous alcohol consumption } \\
\hline No risk & $22(3.4 \%)$ & $623(96.7 \%)$ & 0.040 & $22(3.2 \%)$ & $625(96.8 \%)$ & 0.679 & $62(3.2 \%)$ & $1559(96.9 \%)$ & 0.548 \\
\hline Low risk & $5(1.1 \%)$ & $191(98.9 \%)$ & & $5(1.9 \%)$ & $201(98.1)$ & & 19 (3.0\%) & $472(97.0 \%)$ & \\
\hline Medium risk & $6(9.6 \%)$ & $57(90.4 \%)$ & & $0(0.0 \%)$ & $68(100 \%)$ & & $4(5.3 \%)$ & $146(94.7 \%)$ & \\
\hline High risk & $0(0.0 \%)$ & $12(100 \%)$ & & $1(2.4 \%)$ & $15(97.6 \%)$ & & $1(0.2 \%)$ & $30(99.8 \%)$ & \\
\hline Addiction likely & $1(6.0 \%)$ & $11(94.0 \%)$ & & $0(0.0 \%)$ & $13(100 \%)$ & & $0(0.0 \%)$ & $29(100 \%)$ & \\
\hline \multicolumn{10}{|l|}{ Exposure to ARVs } \\
\hline ARV exposed & & & & & & & $56(3.2 \%)$ & $1331(96.8 \%)$ & 0.675 \\
\hline ARV naive & & & & & & & $26(3.3 \%)$ & $680(96.7 \%)$ & \\
\hline \multicolumn{10}{|l|}{ Viral load suppressed } \\
\hline Yes & & & & & & & $56(3.0 \%)$ & $1430(97.0 \%)$ & 0.392 \\
\hline No & & & & & & & $30(3.5 \%)$ & 790 (96.5\%) & \\
\hline
\end{tabular}


Table 2 Sociodemographic characteristics and diabetes among ALHIV 25 years and older by survey year for 2005, 2008 and 2017 in South Africa (Continued)

\begin{tabular}{|c|c|c|c|c|c|c|c|c|c|}
\hline \multirow[b]{2}{*}{ Population characteristics } & \multicolumn{3}{|l|}{2005} & \multicolumn{3}{|l|}{2008} & \multicolumn{3}{|l|}{2017} \\
\hline & Diabetes & No diabetes & $p$-value & Diabetes & No diabetes & $p$-value & Diabetes & No diabetes & $p$-value \\
\hline \multicolumn{10}{|l|}{ Perception of general health } \\
\hline Excellent & $1(1.5 \%)$ & 92 (98.5\%) & 0.001 & $1(0.9 \%)$ & $165(99.1 \%)$ & 0.031 & $6(1.2 \%)$ & $406(98.8 \%)$ & $<0.001$ \\
\hline Good & $14(1.8 \%)$ & $537(98.2 \%)$ & & $10(2.0 \%)$ & $481(98.0 \%)$ & & $42(2.6 \%)$ & $1278(97.5 \%)$ & \\
\hline Fair & $15(8.4 \%)$ & $214(91.6 \%)$ & & $15(6.2 \%)$ & $223(93.8 \%)$ & & $22(4.1 \%)$ & 457 (95.9\%) & \\
\hline Poor & $4(3.9 \%)$ & $62(96.1 \%)$ & & $3(2.7 \%)$ & $67(97.3 \%)$ & & $17(13.9 \%)$ & 99 (86.1\%) & \\
\hline \multicolumn{10}{|l|}{ Hypertension } \\
\hline Yes & $20(15.6 \%)$ & $103(84.4 \%)$ & $<0.001$ & $17(16.1 \%)$ & $88(83.9 \%)$ & $<0.001$ & $49(11.3 \%)$ & $322(88.7 \%)$ & $<0.001$ \\
\hline No & $14(1.6 \%)$ & $804(98.4 \%)$ & & $10(1.4 \%)$ & $849(98.7 \%)$ & & $35(1.7 \%)$ & 1919 (98.3\%) & \\
\hline \multicolumn{10}{|l|}{ Heart disease } \\
\hline Yes & & & & $5(22.1 \%)$ & $15(77.9 \%)$ & $<0.001$ & $4(5.5 \%)$ & 44 (94.5\%) & 0.298 \\
\hline No & & & & $23(2.4 \%)$ & $920(97.6 \%)$ & & $83(3.1 \%)$ & 2188 (96.9\%) & \\
\hline \multicolumn{10}{|l|}{ Cancer } \\
\hline Yes & $3(54.5 \%)$ & $2(45.5 \%)$ & $<0.001$ & $2(48.7 \%)$ & $4(51.3 \%)$ & $<0.001$ & $3(41.5 \%)$ & $5(58.5 \%)$ & $<0.001$ \\
\hline No & 31 (2.9\%) & 905 (97.1\%) & & $25(2.3 \%)$ & $933(97.7 \%)$ & & 84 (3.1\%) & 2230 (96.9\%) & \\
\hline \multicolumn{10}{|l|}{ Mental distress } \\
\hline Yes & 19 (5.0\%) & 376 (95.0\%) & 0.061 & $3(8.9 \%)$ & 33 (91.1\%) & 0.053 & 25 (3.4\%) & 615 (96.6\%) & 0.789 \\
\hline No & $15(2.1 \%)$ & 527 (97.9\%) & & $24(2.3 \%)$ & 902 (97.7\%) & & $62(3.1 \%)$ & 1627 (96.9\%) & \\
\hline \multicolumn{10}{|l|}{ Tuberculosis } \\
\hline Yes & $4(7.4 \%)$ & 57 (92.6\%) & 0.134 & $4(7.1 \%)$ & 45 (92.9\%) & 0.116 & 8 (5.9\%) & 68 (94.1\%) & 0.142 \\
\hline No & $30(3.0 \%)$ & 850 (97.0\%) & & $24(2.5 \%)$ & 892 (95.5\%) & & 79 (3.1\%) & 2168 (96.9\%) & \\
\hline \multicolumn{10}{|l|}{ Health care access } \\
\hline Public & $28(3.2 \%)$ & 753 (96.8\%) & 0.559 & $21(2.3 \%)$ & 719 (97.7\%) & 0.190 & $69(2.8 \%)$ & 2034 (97.2\%) & 0.002 \\
\hline Private & $6(4.4 \%)$ & 130 (95.6\%) & & $7(5.1 \%)$ & 193 (94.9\%) & & 17 (8.1\%) & 184 (91.9\%) & \\
\hline
\end{tabular}

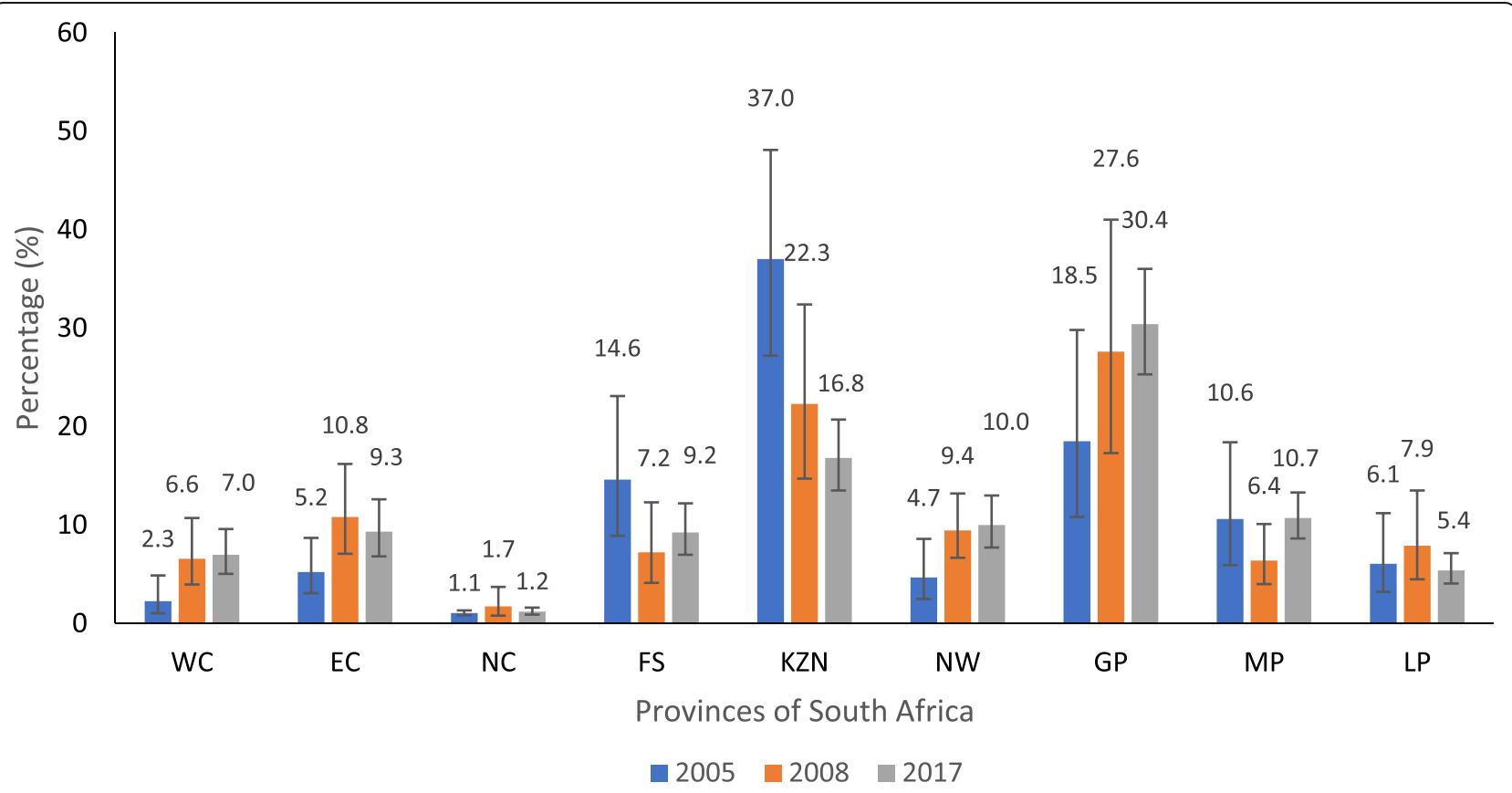

Fig. 1 Prevalence of hypertension among ALHIV by provinces in 2005, 2008 \& 2017 


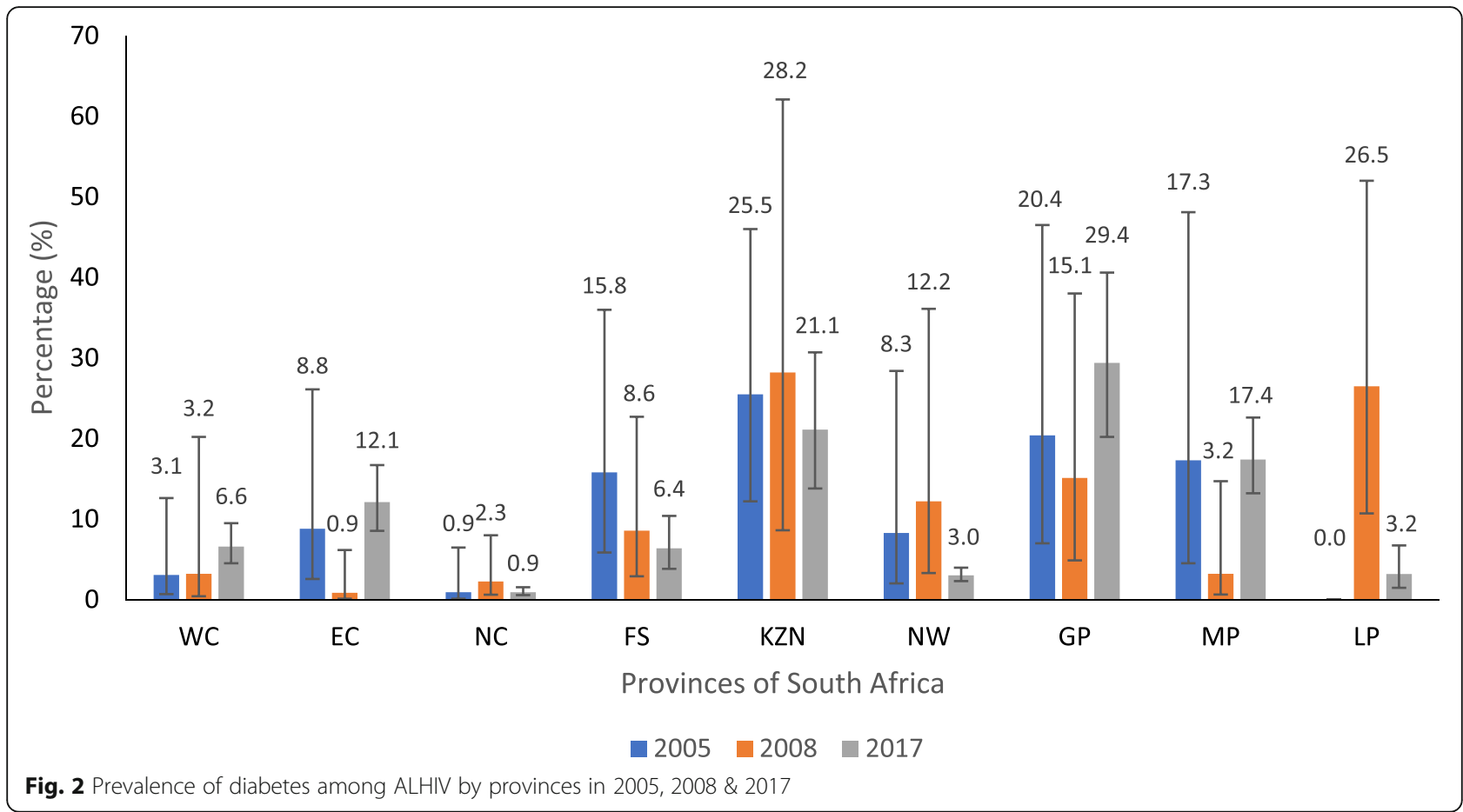

Table 3 Factors associated with hypertension for 2005

\begin{tabular}{|c|c|c|}
\hline Variables in the model & Crude OR (95\% Cl) & Adjusted OR $(95 \% \mathrm{Cl})$ \\
\hline \multicolumn{3}{|l|}{ Sex } \\
\hline Male & Reference & Reference \\
\hline Female & $1.81(0.98-3.37)$ & $2.59(1.26-5.32) *$ \\
\hline \multicolumn{3}{|l|}{ Age groups } \\
\hline 25-34 years & Reference & Reference \\
\hline $35-44$ years & $1.46(0.73-2.92)$ & $1.72(0.81-3.64)$ \\
\hline $45+$ years & $2.90(1.43-5.89) * *$ & $4.03(1.96-8.28) * * *$ \\
\hline \multicolumn{3}{|l|}{ Race } \\
\hline Non-Black African & Reference & Reference \\
\hline Black African & $1.15(0.47-2.85)$ & $0.96(0.34-2.81)$ \\
\hline \multicolumn{3}{|c|}{ Hazardous alcohol consumption } \\
\hline No risk & Reference & Reference \\
\hline Low risk & $0.47(0.23-0.95) *$ & $0.56(0.26-1.24)$ \\
\hline Medium risk & $1.28(0.55-2.98)$ & $1.48(0.60-3.69)$ \\
\hline High risk & $0.38(0.05-3.18)$ & $0.38(0.04-3.37)$ \\
\hline Addiction likely & $0.29(0.03-2.44)$ & $0.18(0.01-2.92)$ \\
\hline \multicolumn{3}{|l|}{ Diabetes } \\
\hline No & Reference & Reference \\
\hline Yes & $11.16(4.14-30.05)^{* * *}$ & $11.73(4.02-34.20) * * *$ \\
\hline \multicolumn{3}{|l|}{ Mental distress } \\
\hline No & Reference & Reference \\
\hline Yes & $2.73(1.54-4.85) * *$ & $2.97(1.59-5.54) * *$ \\
\hline
\end{tabular}

migration and a rise in the elderly population which may be attributed to ART in PLHIV [40].

Our study also found that females, aged 45 years and older, and those who resided in an urban area had the highest prevalence of both NCDs for all 3 years. The high prevalence in women may be as a result of an underestimation in men reflecting more on their healthseeking behaviour than disease prevalence itself $[14,18$, $34,35,41,42]$. The impact of urbanisation has also been documented in studies such as the 2012 SANHANES study and the South African cross-sectional study on HIV positive educators which suggest that urban living leads to easy access to unhealthy diets and a sedentary lifestyle [34, 35]. In 2008 and 2017, we further see that those who had primary education and below also had a high prevalence of hypertension and diabetes as supported by Ntuli et al. [43]. Wang et al. suggest that not only an unhealthy diet but even a lack of regular and effective physical exercise and higher alcohol intake can be correlated with low levels of schooling [44]. The presence of comorbidities was also evident with higher proportions of individuals with cancer, heart disease, TB and mental distress reporting having hypertension and diabetes. This is consistent with studies which observed similar findings globally and in Africa [34, 45-47]. This could mean that PLHIV who have a comorbidity require close monitoring to ensure that their health conditions are effectively managed thereby reducing the risk of acquiring more NCDs. 
Table 4 Factors associated with hypertension for 2008

\begin{tabular}{|c|c|c|}
\hline Variables in the model & Crude OR (95\% Cl) & Adjusted OR (95\% Cl) \\
\hline \multicolumn{3}{|l|}{ Sex } \\
\hline Male & Reference & Reference \\
\hline Female & $4.81(2.10-11.02)^{* * *}$ & $4.31(1.94-9.58) * * *$ \\
\hline \multicolumn{3}{|l|}{ Age groups } \\
\hline 25-34 years & Reference & Reference \\
\hline $35-44$ years & $1.68(0.76-3.71)$ & $2.22(0.99-4.97)$ \\
\hline $45+$ years & $6.95(3.34-14.45) * * *$ & $8.44(3.92-18.15)^{* * *}$ \\
\hline \multicolumn{3}{|l|}{ Race } \\
\hline Non-African & Reference & Reference \\
\hline African & $0.65(0.19-2.17)$ & $0.67(0.14-3.28)$ \\
\hline \multicolumn{3}{|l|}{ Locality } \\
\hline Tribal area/Rural informal & Reference & Reference \\
\hline Rural formal & $0.96(0.25-3.66)$ & $0.93(0.35-2.46)$ \\
\hline Urban informal & $1.77(0.90-3.51)$ & $2.40(1.06-5.43) *$ \\
\hline Urban formal & $2.20(1.13-4.28) *$ & $2.95(1.34-6.53) * *$ \\
\hline \multicolumn{3}{|c|}{ Hazardous alcohol consumption } \\
\hline No risk & Reference & Reference \\
\hline Low risk & $1.20(0.61-2.36)$ & $1.44(0.66-3.12)$ \\
\hline Medium risk & $0.50(.018-1.42)$ & $0.78(0.26-2.35)$ \\
\hline High risk & $0.11(0.01-0.97) *$ & $0.15(0.01-1.78)$ \\
\hline Addiction likely & $0.16(0.02-1.47)$ & $0.25(0.03-1.97)$ \\
\hline \multicolumn{3}{|l|}{ Perception of general health } \\
\hline Excellent & Reference & Reference \\
\hline Good & $4.92(1.49-16.22) * *$ & $3.72(1.00-13.81) *$ \\
\hline Fair & $7.56(2.33-24.58) * *$ & $5.44(1.40-21.12) *$ \\
\hline Poor & $4.95(1.30-18.80) *$ & $4.82(1.05-22.20) *$ \\
\hline \multicolumn{3}{|l|}{ Diabetes } \\
\hline No & Reference & Reference \\
\hline Yes & $14.03(5.27-37.35) * * *$ & $6.16(1.68-22.59){ }^{* *}$ \\
\hline \multicolumn{3}{|l|}{ Heart disease } \\
\hline No & Reference & Reference \\
\hline Yes & $4.26(1.33-13.70) *$ & $1.13(0.23-5.52)$ \\
\hline \multicolumn{3}{|l|}{ Health care access } \\
\hline Private & Reference & Reference \\
\hline Public & $1.76(0.88-3.51)$ & $2.58(1.02-6.51) *$ \\
\hline
\end{tabular}

Factors that increased the odds of hypertension for all 3 years in our study included being female, aged 45 years and older, or having diabetes. Antonello et al., Malaza et al., and Zungu et al. suggest that increased odds in females were mainly attributed to women having higher BMI's and hip to weight ratios than men and women being more physically inactive, all of which are considered risk factors [35, 41, 48]. Ageing has also been well described to result in gradual vascular stiffening and the prolonged use of ART particularly protease inhibitors is related to the production of vascular reactive oxygen species resulting in hypertension [17, 23, 24]. While diabetes has been suggested by Petrie et al., and Gazzaruso et al. to result from hypertension-linked insulin resistance due to multiple pathophysiological mechanisms influencing microvascular and endothelial functioning [24, 25]. Mental distress also increased the odds of having high blood pressure in 2005. An American study found 
Table 5 Factors associated with hypertension for 2017

\begin{tabular}{|c|c|c|}
\hline Variables in the model & Crude OR $(95 \% \mathrm{Cl})$ & Adjusted OR (95\% Cl) \\
\hline \multicolumn{3}{|l|}{ Sex } \\
\hline Male & Reference & Reference \\
\hline Female & $1.76(1.29-2.41) * * *$ & $2.33(1.60-3.42) * * *$ \\
\hline \multicolumn{3}{|l|}{ Age groups } \\
\hline $25-34$ years & Reference & Reference \\
\hline $35-44$ years & $1.78(1.11-2.83) *$ & $1.83(1.12-3.00) *$ \\
\hline $45+$ years & $7.24(4.88-10.74) * * *$ & $7.32(4.78-11.21)^{* * *}$ \\
\hline \multicolumn{3}{|l|}{ Race } \\
\hline Non-Black African & Reference & Reference \\
\hline Black African & $0.65(0.39-1.07)$ & $0.61(0.33-1.13)$ \\
\hline \multicolumn{3}{|l|}{ Employment status } \\
\hline Unemployed & Reference & Reference \\
\hline Employed & $0.68(0.48-0.98) *$ & $0.76(0.50-1.15)$ \\
\hline Other (incl. Old age pensioner, sick/disabled and unable to work) & $2.80(1.45-5.40) * *$ & $2.27(1.09-4.73) *$ \\
\hline \multicolumn{3}{|l|}{ Locality } \\
\hline Rural informal & Reference & Reference \\
\hline Rural (farms) & $0.85(0.51-1.42)$ & $1.58(0.86-2.90)$ \\
\hline Urban & $1.44(1.08-1.91) *$ & $1.61(1.16-2.23) * *$ \\
\hline \multicolumn{3}{|l|}{ Exposure to ARVs } \\
\hline ARV naive & Reference & Reference \\
\hline ARV exposed & $1.20(0.85-1.68)$ & $0.97(0.67-1.40)$ \\
\hline \multicolumn{3}{|l|}{ Hazardous alcohol consumption } \\
\hline No risk & Reference & Reference \\
\hline Low risk & $1.23(0.88-1.71)$ & $1.26(0.83-1.93)$ \\
\hline Medium risk & $0.47(0.24-0.91) *$ & $0.61(0.29-1.30)$ \\
\hline High risk & $1.78(0.57-5.57)$ & $4.43(1.67-11.76) * *$ \\
\hline Addiction likely & $0.78(0.31-1.94)$ & $1.10(0.43-2.78)$ \\
\hline \multicolumn{3}{|l|}{ Diabetes } \\
\hline No & Reference & Reference \\
\hline Yes & $7.38(4.41-12.35) * * *$ & $5.17(2.69-9.96) * * *$ \\
\hline \multicolumn{3}{|l|}{ Heart disease } \\
\hline No & Reference & Reference \\
\hline Yes & $4.57(2.35-8.88) * * *$ & $3.36(1.59-7.10) * *$ \\
\hline
\end{tabular}

${ }^{*} p<0.05,{ }^{* *} p<0.01,{ }^{* * *} p<0.001$

that one's mental health affects one's ability to maintain a healthy lifestyle, seek early treatment for comorbid conditions, or consistently adhere to treatment programs [45]. Living in an urban area was another significant factor associated with hypertension in 2008 and 2017. Findings from previous studies suggest that urbanisation and the associated lifestyle change including poor diet and low physical activity are risk factors of hypertension [49]. While having a high risk of hazardous alcohol consumption or having heart disease were also additional associated factors for hypertension found in 2017. In
Zimbabwean and Brazilian studies alcohol consumption increased the risk of hypertension, however, there have been several hypotheses proposed for this association $[50,51]$. Some researchers have to an extent been able to characterize the pathogenesis due to harmful alcohol drinking, citing that a reduction in vasodilators like nitric oxide occurs leading to an inflammatory lack of relaxation and oxidative injury of the blood vessel lining [52]. Other researchers have suggested the link between harmful alcohol consumption and the eventual accumulation of triglycerides and total cholesterol, both of 
Table 6 Factors associated with diabetes for 2005

\begin{tabular}{|c|c|c|}
\hline Variables in the model & Crude OR $(95 \% \mathrm{Cl})$ & Adjusted OR $(95 \% \mathrm{Cl})$ \\
\hline \multicolumn{3}{|l|}{ Sex } \\
\hline Male & Reference & Reference \\
\hline Female & $0.55(0.22-1.39)$ & $0.41(0.14-1.20)$ \\
\hline \multicolumn{3}{|l|}{ Age groups } \\
\hline $25-34$ years & Reference & Reference \\
\hline $35-44$ years & $1.00(0.35-2.89)$ & $0.88(0.29-2.66)$ \\
\hline $45+$ years & $1.32(0.44-3.93)$ & $0.58(0.19-7.75)$ \\
\hline \multicolumn{3}{|l|}{ Race } \\
\hline Non-Black African & Reference & Reference \\
\hline Black African & $3.77(1.04-13.65) *$ & $5.70(1.10-29.44) *$ \\
\hline \multicolumn{3}{|c|}{ Perception of general health } \\
\hline Excellent & Reference & Reference \\
\hline Good & $1.20(0.15-9.78)$ & $1.41(0.26-7.62)$ \\
\hline Fair & $5.91(0.71-48.97)$ & $5.16(0.92-29.01)$ \\
\hline Poor & $2.61(0.25-27.00)$ & $2.30(0.33-16.05)$ \\
\hline \multicolumn{3}{|l|}{ Hypertension } \\
\hline No & Reference & Reference \\
\hline Yes & $11.16(4.14-30.05)^{* * *}$ & $11.71(3.78-36.29) * * *$ \\
\hline \multicolumn{3}{|l|}{ Mental distress } \\
\hline No & Reference & Reference \\
\hline Yes & $2.47(0.93-6.54)$ & $1.09(0.40-2.92)$ \\
\hline \multicolumn{3}{|l|}{ Health facility of choice } \\
\hline Private & Reference & Reference \\
\hline Public & $0.73(0.25-2.14)$ & $0.42(0.15-1.11)$ \\
\hline
\end{tabular}

Table 7 Factors associated with diabetes for 2008

\begin{tabular}{lll}
\hline Variables in the model & Crude OR $\mathbf{( 9 5 \% ~ C l )}$ & Adjusted OR $\mathbf{( 9 5 \% ~ C l )}$ \\
\hline Sex & & \\
Male & Reference & Reference \\
Female & $2.31(0.57-9.32)$ & $1.28(0.28-5.77)$
\end{tabular}

Age groups

$\begin{array}{cll}\text { 25-34 years } & \text { Reference } & \text { Reference } \\ \text { 35-44 years } & 2.03(0.46-8.98) & 1.95(0.43-8.86) \\ 45+\text { years } & 9.14(2.67-31.27) * * & 4.44(1.00-19.73) * \\ \text { Race } & & \\ \text { Non-African } & \text { Reference } & \text { Reference } \\ \text { African } & 0.32(0.08-1.35) & 0.39(0.09-1.81)\end{array}$

Hypertension

$\begin{array}{lll}\text { No } & \text { Reference } & \text { Reference } \\ \text { Yes } & 14.03(5.27-37.35) * * * & 7.56(2.29-24.92) * * *\end{array}$

Heart disease

\begin{tabular}{ccl} 
No & Reference & Reference \\
Yes & $11.71(3.02-45.39) * * *$ & $6.92(1.62-29.62) * *$ \\
\hline${ }^{*} p<0.05,{ }^{* *} p<0.01,{ }^{* * *} p<0.001$ &
\end{tabular}

Table 8 Factors associated with diabetes for 2017

\begin{tabular}{|c|c|c|}
\hline Variables in the model & Crude OR (95\% Cl) & Adjusted OR (95\% Cl) \\
\hline \multicolumn{3}{|l|}{ Sex } \\
\hline Male & Reference & Reference \\
\hline Female & $1.47(0.80-2.70)$ & $1.80(0.86-3.78)$ \\
\hline \multicolumn{3}{|l|}{ Age groups } \\
\hline $25-34$ years & Reference & Reference \\
\hline $35-44$ years & $3.88(1.01-14.93) *$ & $6.38(1.80-22.62){ }^{* *}$ \\
\hline $45+$ years & $9.88(2.96-32.95) * * *$ & $7.90(2.11-29.58) * *$ \\
\hline \multicolumn{3}{|l|}{ Race } \\
\hline Non-African & Reference & Reference \\
\hline African & $0.54(0.211-1.36)$ & $0.52(0.15-1.82)$ \\
\hline \multicolumn{3}{|l|}{ Educational level } \\
\hline None or Primary & Reference & Reference \\
\hline Secondary and above & $0.22(0.13-0.39)^{* * *}$ & $0.31(0.16-0.59) * * *$ \\
\hline \multicolumn{3}{|l|}{ Exposure to ARVs } \\
\hline ARV naive & Reference & Reference \\
\hline ARV exposed & $0.98(0.54-1.77)$ & $0.94(0.47-1.85)$ \\
\hline \multicolumn{3}{|c|}{ Perception of general health } \\
\hline Excellent & Reference & Reference \\
\hline Good & $2.18(0.86-5.52)$ & $1.15(0.41-3.26)$ \\
\hline Fair & $3.56(1.30-9.81) *$ & $1.04(0.32-3.35)$ \\
\hline Poor & $13.46(4.63-39.15) * * *$ & $6.48(1.65-25.41)^{* *}$ \\
\hline \multicolumn{3}{|l|}{ Hypertension } \\
\hline No & Reference & Reference \\
\hline Yes & $7.38(4.41-12.35) * * *$ & $4.60(2.34-9.07) * * *$ \\
\hline \multicolumn{3}{|l|}{ Heart disease } \\
\hline No & Reference & Reference \\
\hline Yes & $1.79(0.59-5.46)$ & $0.77(0.18-3.31)$ \\
\hline \multicolumn{3}{|l|}{ Healthcare access } \\
\hline Private & Reference & Reference \\
\hline Public & $0.34(0.17-0.70) * *$ & $0.25(0.11-0.60) * *$ \\
\hline
\end{tabular}

which have been documented to have a link to hypertension [53]. In addition, according to the WHO, heart disease can result from hypertension in the general population [54]. Hypertension and diabetes have long been documented to be a major modifiable risk factor for heart disease due to the progressive damage to the heart that occurs over time $[55,56]$. Lastly, in 2017, the employment category "other" which included pensioners, sick and disabled people, was also associated with hypertension. The reasoning for this includes that pensioners are presumably older hence the effect of age may have attributed to this finding; also, sick and disabled people are more likely to be non-mobile and be physically inactive both of which again are 
characteristics that have been documented to increase the risk of hypertension.

The common significant factor associated with diabetes across the years was having hypertension and the supporting reasons and studies are identical to those mentioned above. In 2008 other additional factors included being 45 years and above and having heart disease; both established risk factors in other studies [49]. In 2017, being 45 years and above, and reporting poor health was significantly associated with diabetes in the study population. Also, having secondary and above education level lowered one's odds of having diabetes. Other studies with a similar finding reported that education increased awareness and therefore the individuals were more likely to make better health-related decisions [18]. The above findings from our study, thus, offer various avenues for possible interventions which may include active awareness campaigns targeted at men, those who have educational qualifications below secondary level or those residing in urban areas. Also, as the HIV population is ageing, closely monitoring the impact of antiretroviral medication could be considered. Lastly, these findings could allow for the refinement of the integrated chronic care model allowing for increased case finding.

\section{Limitations}

This study had a few limitations. Due to the crosssectional nature of the surveys, causality cannot be determined in the study population. Questionnaire data used were self-reported and therefore prone to social desirability, recall bias and underreporting. The analysis did not account for unmeasured and unreported risk factors and other confounders that may have had an impact on the outcome variables. Also, as hypertension and diabetes were not measured or diagnostically determined, misclassification bias may have been introduced. Lastly, wide confidence intervals for some variables were observed and this may have been as a result of a small sample answering particular questions, therefore, conclusions drawn from the data need to be replicated with a larger sample size. Nevertheless, the surveys were nationally representative and can be generalised to adults aged 25 years and above living with HIV with hypertension and diabetes.

\section{Conclusion}

Population-based nationally representative surveys are important for developing sound health decisions and policies that would affect the country. This study identified factors consistently associated with the prevalence of hypertension and diabetes among ALHIV overtime. Additional contemporary risk factors were also identified. Therefore, these findings contribute to understanding the trend and identifying modifiable factors responsible for hypertension and diabetes among ALHIV adults from 2005 to 2017. This may help inform the development of integrated chronic disease management model to reduce the co-morbid burden of disease and associated adverse health outcomes.

\section{Abbreviations}

ALHIV: Adults living with HIV; ART: Antiretroviral Therapy; AUDIT: Alcohol Use Disorder Identification Test; CDC: Centers for Disease Control and Prevention; Cl: Confidence Interval; HIV: Human immunodeficiency virus; HSRC: Human Sciences Research Council; IDF: International Diabetes Federation;

NCDs: Non-communicable diseases; OR: Odds ratios; PLHIV: People Living with HIV; REC: Research Ethics Committee; SABSSM: South African National HIV Prevalence, Incidence, Behaviour and Communication survey; SANHAN ES: South African National Health and Nutrition Examination Survey; WHO: World Health Organization

\section{Supplementary Information}

The online version contains supplementary material available at https://doi. org/10.1186/s12889-021-10502-8.

Additional file 1: Table S1. Characteristics of adults living with HIV in South Africa: 2005, 2008 \& 2017. Figure S1. Distribution of study participants by province in 2005, 2008 \& 2017.

\section{Acknowledgements}

Not applicable.

\section{Authors' contributions}

NC, NZ and CC conceptualized the study. NC, MM and NZ contributed to the methodology. NC did the formal analysis. NZ and CC supervised the study. NC wrote the original draft. All authors reviewed, edited and approved the final manuscript.

Funding

Not applicable.

\section{Availability of data and materials}

The dataset(s) are accessible on request on the HSRC data research repository http://datacuration.hsrc.ac.za/.

\section{Declarations}

Ethics approval and consent to participate

Ethical clearance for the secondary analysis was obtained from the University of the Witwatersrand Human Research Ethics Committee (certificate M191181). Ethical approval for the primary studies was obtained from the HSRC Research Ethics Committee (2005 certificate REC: 5/24/05/04; 2008 certificate REC: 2/23/10/07; 2017 certificate REC: 4/18/11/15) and the USA's Centers for Disease Control and Prevention (CDC). Voluntary informed written consent was submitted by all individuals who agreed to participate in the studies. All methods were performed in accordance with the relevant guidelines and regulations.

Consent for publication

Not applicable.

Competing interests

The authors declare that they have no competing interests.

\section{Author details}

${ }^{1}$ Division of Epidemiology and Biostatistics, School of Public Health, Faculty of Health Sciences, University of the Witwatersrand, Johannesburg, South Africa. ${ }^{2}$ Human Sciences Research Council, Pretoria, South Africa.

${ }^{3}$ Department of Psychology, University of Pretoria, Pretoria, South Africa. ${ }^{4}$ Implementation Science Unit Programme, Right to Care, Johannesburg, South Africa. 
Received: 27 December 2020 Accepted: 24 February 2021 Published online: 06 March 2021

\section{References}

1. Simbayi LC, Zuma K, Zungu N, Moyo S, Marinda E, Jooste S, et al. South African national HIV prevalence, incidence, behaviour and communication survey, 2017. Cape Town: HSRC Press; 2019.

2. Joint United Nations Programme on HIV/AIDS. HIV estimates with uncertainty bounds 1990-2019 Geneva, 2020 [05/11/2020]. Available from: https://www.unaids.org/en/resources/documents/2020/HIV_estimates_with_ uncertainty bounds 1990-present

3. Evans D. Ten years on ART - where to now? 2013.

4. Haal K, Smith A, van Doorslaer E. The rise and fall of mortality inequality in South Africa in the HIV era. SSM Popul Health. 2018;5:239-48.

5. Joint United Nations Programme on HIV/AIDS. Chronic care of HIV and noncommunicable diseases: how to leverage the HIV experience. Geneva: UNAIDS; 2011.

6. Patel P, Rose CE, Collins PY, Nuche-Berenguer B, Sahasrabuddhe W, Peprah E, et al. Noncommunicable diseases among HIV-infected persons in lowincome and middle-income countries: a systematic review and metaanalysis. AIDS. 2018;32(Suppl 1):S5-S20.

7. Kamkuemah M, Gausi B, Oni T. Missed opportunities for NCD multimorbidity prevention in adolescents and youth living with HIV in urban South Africa. BMC Public Health. 2020;20(1):821

8. Tollman SM, Kahn K, Sartorius B, Collinson MA, Clark SJ, Garenne ML. Implications of mortality transition for primary health care in rural South Africa: a population-based surveillance study. Lancet. 2008;372(9642):893901.

9. Erzse A, Stacey N, Chola L, Tugendhaft A, Freeman M, Hofman K. The direct medical cost of type 2 diabetes mellitus in South Africa: a cost of illness study. Glob Health Action. 2019;12(1):1636611.

10. Gaziano TA, Steyn K, Cohen DJ, Weinstein MC, Opie LH. Cost-effectiveness analysis of hypertension guidelines in South Africa. Circulation. 2005;112(23): 3569-76.

11. Todowede OO, Mianda SZ, Sartorius B. Prevalence of metabolic syndrome among HIV-positive and HIV-negative populations in sub-Saharan Africa-a systematic review and meta-analysis. Syst Rev. 2019;8(1):4

12. Ataro Z, Ashenafi W, Fayera J, Abdosh T. Magnitude and associated factors of diabetes mellitus and hypertension among adult HIV-positive individuals receiving highly active antiretroviral therapy at Jugal Hospital, Harar, Ethiopia. Hiv Aids (Auckl). 2018;10:181-92.

13. Ekrikpo UE, Akpan EE, Ekott JU, Bello AK, Okpechi IG, Kengne AP. Prevalence and correlates of traditional risk factors for cardiovascular disease in a Nigerian ART-naive HIV population: a cross-sectional study. BMJ Open. 2018; 8(7):e019664.

14. Magodoro IM, Esterhuizen TM, Chivese T. A cross-sectional, facility based study of comorbid non-communicable diseases among adults living with HIV infection in Zimbabwe. BMC Res Notes. 2016:9:379.

15. Bailey SL, Ayles H, Beyers N, Godfrey-Faussett P, Muyoyeta M, du Toit E, et al. Diabetes mellitus in Zambia and the Western cape province of South Africa: prevalence, risk factors, diagnosis and management. Diabetes Res Clin Pract. 2016;118:1-11.

16. Gbadamosi MA, Tlou B. Prevalence of abnormal glucose metabolism among adults attending an outpatient department at a tertiary referral hospital in Swaziland: a cross-sectional study. BMC Public Health. 2020;20(1):392.

17. Peck RN, Shedafa R, Kalluvya S, Downs JA, Todd J, Suthanthiran M, et al. Hypertension, kidney disease, HIV and antiretroviral therapy among Tanzanian adults: a cross-sectional study. BMC Med. 2014;12(1):125.

18. Kwarisiima D, Balzer L, Heller D, Kotwani P, Chamie G, Clark T, et al. Population-Based Assessment of Hypertension Epidemiology and Risk Factors among HIV-Positive and General Populations in Rural Uganda. PLoS One. 2016;11(5):e0156309.

19. Magula NP, Motala AA, Lalloo UG. The prevalence and incidence of diabetes mellitus and other disorders of glycaemia in south African black patients on antiretroviral therapy. Int J Infect Dis. 2014:21:10.

20. Prioreschi A, Munthali RJ, Soepnel L, Goldstein JA, Micklesfield LK, Aronoff DM, et al. Incidence and prevalence of type 2 diabetes mellitus with HIV infection in Africa: a systematic review and meta-analysis. BMJ Open. 2017; 7(3):e013953.

21. Pinto E. Blood pressure and ageing. Postgrad Med J. 2007:83(976):109-14.
22. Kirkman MS, Briscoe VJ, Clark N, Florez H, Haas LB, Halter JB, et al. Diabetes in Older Adults. Diabetes Care. 2012;35(12):2650-64.

23. Fahme SA, Bloomfield GS, Peck R. Hypertension in HIV-infected adults: novel pathophysiologic mechanisms. Hypertension. 2018;72(1):44-55.

24. Petrie JR, Guzik TJ, Touyz RM. Diabetes, hypertension, and cardiovascular disease: clinical insights and vascular mechanisms. Can J Cardiol. 2018;34(5): 575-84.

25. Gazzaruso C, Bruno R, Garzaniti A, Giordanetti S, Fratino P, Sacchi $P$, et al. Hypertension among HIV patients: prevalence and relationships to insulin resistance and metabolic syndrome. J Hypertens. 2003;21(7):1377-82.

26. Mutyambizi C, Chola L, Groot W, Pavlova M, Labadarios D, Hongoro C. The extent and determinants of diabetes and cardiovascular disease comorbidity in South Africa - results from the south African National Health and nutrition examination survey (SANHANES-1). BMC Public Health. 2017; 17(1):745.

27. Divala $\mathrm{OH}$, Amberbir A, Ismail Z, Beyene T, Garone D, Pfaff C, et al. The burden of hypertension, diabetes mellitus, and cardiovascular risk factors among adult Malawians in HIV care: consequences for integrated services. BMC Public Health. 2016;16(1):1243.

28. Shisana ORT, Simbayi LC, Parker W, Zuma K, Bhana A, Connolly C, Jooste S, Pillay V, et al. South African National HIV Prevalence, Incidence, Behaviour and Communication Survey, 2005. Cape Town: HSRC Press; 2005.

29. Shisana O, Rehle T, Simbayi LC, Zuma K, Jooste S, Pillay-van-Wyk V, et al. South African national HIV prevalence, incidence, behaviour and communication survey 2008: a turning tide among teenagers? Cape Town: HSRC Press; 2009.

30. Shisana O, Rehle T, Simbayi LC, Zuma K, Jooste S, Zungu N, et al. South African national HIV prevalence, incidence and behaviour survey, 2012. 2014.

31. Reid MC, Fiellin DA, O'Connor PG. Hazardous and harmful alcohol consumption in primary care. Arch Intern Med. 1999;159(15):1681-9.

32. StataCorp. Stata Statistical Software: Release 15. College Station: StataCorp LLC; 2017.

33. Zhang Z. Model building strategy for logistic regression: purposeful selection. Ann Transl Med. 2016:4(6):111.

34. Shisana O, Labadarios D, Rehle T, Simbayi L, Zuma K, Dhansay A, et al. The south African National Health and nutrition examination survey, 2012: SANH ANES-1: the health and nutritional status of the nation. 2014

35. Zungu NP, Mabaso ML, Kumalo F, Sigida S, Mlangeni L, Wabiri N, et al. Prevalence of non-communicable diseases (NCDs) and associated factors among HIV positive educators: findings from the 2015/6 survey of health of educators in public schools in South Africa. PLoS One. 2019;14(2):e0209756.

36. van Heerden A, Barnabas RV, Norris SA, Micklesfield LK, van Rooyen H, Celum C. High prevalence of HIV and non-communicable disease (NCD) risk factors in rural KwaZulu-Natal, South Africa. J Int AIDS Soc. 2017;20(2): e25012.

37. Chu C, Umanski G, Blank A, Meissner P, Grossberg R, Selwyn PA. Comorbidity-related treatment outcomes among HIV-infected adults in the Bronx. NY J Urban Health. 2011;88(3):507-16.

38. International Diabetes Federation. IDF Diabetes Atlas, 9th edn Brussels, Belgium, 2019 [07/08/2020]. Available from: https://www.diabetesatlas.org.

39. Knowledge Translation Unit UoCTLI. Adult primary care guide (APC) 2019/ 2020. Pretoria: The National Department of Health of South Africa; 2019.

40. StatsSA. Statistics South Africa. Formal census. 2011.

41. Antonello VS. Carlos Ferreira Antonello I, Grossmann TK, Tovo CV, Brasil dal Pupo B, de Quadros Winckler L. hypertension—an emerging cardiovascular risk factor in HIV infection. J Am Soc Hypertens. 2015;9(5):403-7.

42. da Cunha GH, Franco KB, Galvão MTG, Lima MAC, Fontenele MSM, Siqueira $L R$, et al. Diabetes mellitus in people living with HIV/AIDS: prevalence and associated risk factors. AIDS Care. 2020;32(5):600-7.

43. Ntuli ST, Maimela E, Alberts M, Choma S, Dikotope S. Prevalence and associated risk factors of hypertension amongst adults in a rural community of Limpopo Province, South Africa. Afr J Prim Health Care Fam Med. 2015: 7(1):847.

44. Wang Y, Chen J, Wang K, Edwards CL. Education as an important risk factor for the prevalence of hypertension and elevated blood pressure in Chinese men and women. J Hum Hypertens. 2006;20(11):898-900.

45. Ojike N, Sowers JR, Seixas A, Ravenell J, Rodriguez-Figueroa G, Awadallah M, et al. Psychological distress and hypertension: results from the National Health Interview Survey for 2004-2013. Cardiorenal Med. 2016;6(3):198-208. 
46. Schutte AE, Ware $\amalg$, Huisman HW, Fourie CMT, Greeff M, Khumalo T, et al. Psychological distress and the development of hypertension over 5 years in black south Africans. J Clin Hypertens. 2015;17(2):126-33.

47. World Health Organization. Global report on diabetes, vol. 2016. Geneva: World Health Organization; 2016.

48. Malaza A, Mossong J, Bärnighausen T. Newell M-L. Hypertension and obesity in adults living in a high HIV prevalence rural area in South Africa. PLoS One. 2012;7(10):e47761.

49. Price AJ, Crampin AC, Amberbir A, Kayuni-Chihana N, Musicha C, Tafatatha $T$, et al. Prevalence of obesity, hypertension, and diabetes, and cascade of care in sub-Saharan Africa: a cross-sectional, population-based study in rural and urban Malawi. Lancet Diabetes Endocrinol. 2018;6(3):208-22.

50. Chireshe R, Naidoo K, Nyamakura R. Hypertension among human immunodeficiency virus infected patients on treatment at Parirenyatwa hospital: a descriptive study. Afr J Prim Health Care Fam Med. 2019;11(1):e18.

51. Ikeda MLR, Barcellos NT, Alencastro PR, Wolff FH, Moreira LB, Gus M, et al. Alcohol drinking pattern: a comparison between HIV-infected patients and individuals from the general population. PLoS One. 2016;11(6):e0158535.

52. Husain K, Ferder L, Ansari RA, Lalla J. Chronic ethanol ingestion induces aortic inflammation/oxidative endothelial injury and hypertension in rats. Hum Exp Toxicol. 2011:30(8):930-9.

53. Hao G, Wang Z, Zhang L, Chen Z, Wang X, Guo M, et al. Relationship between alcohol consumption and serum lipid profiles among middle-aged population in China: a multiple-center cardiovascular epidemiological study. Angiology. 2015;66(8):753-8.

54. World Health Organisation. Fact Sheet - Hypertension, 2019 Accessed 19 January 2020. Available from: https://www.who.int/news-room/fact-sheets/ detail/hypertension,

55. Fuchs FD, Whelton PK. High blood pressure and cardiovascular disease. Hypertension. 2020;75(2):285-92.

56. Leon BM, Maddox TM. Diabetes and cardiovascular disease: epidemiology, biological mechanisms, treatment recommendations and future research. World J Diabetes. 2015;6(13):1246-58.

\section{Publisher's Note}

Springer Nature remains neutral with regard to jurisdictional claims in published maps and institutional affiliations.

Ready to submit your research? Choose BMC and benefit from:

- fast, convenient online submission

- thorough peer review by experienced researchers in your field

- rapid publication on acceptance

- support for research data, including large and complex data types

- gold Open Access which fosters wider collaboration and increased citations

- maximum visibility for your research: over $100 \mathrm{M}$ website views per year

At $\mathrm{BMC}$, research is always in progress.

Learn more biomedcentral.com/submissions 